\title{
Capacitance-voltage spectroscopy and analysis of dielectric intrinsic amorphous silicon thin films
}

\author{
Sebastian Gerke ${ }^{*}, 1$, Gabriel Micard", Reinhart Job², Giso Hahn ${ }^{1}$, and Barbara Terheiden ${ }^{1}$ \\ ${ }^{1}$ Department of Physics, University of Konstanz, 78457 Konstanz, Germany \\ ${ }^{2}$ Department of Electrical Engineering and Computer Science, Münster University of Applied Sciences, 48565 Steinfurt, Germany
}

Keywords amorphous silicon (a-Si), dangling bonds, capacitance-voltage (CV) spectroscopy

* Corresponding author: e-mail sebastian.gerke@uni-konstanz.de, Phone: +49 753188 213 2, Fax: +49 7531883895

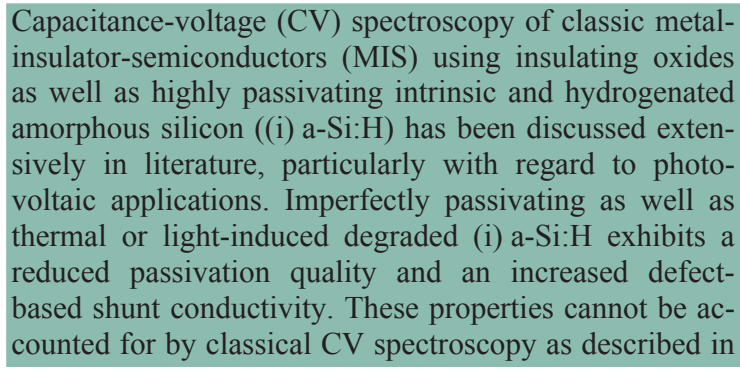

1 Introduction Capacitance-voltage (CV) spectroscopy of a metal-insulator-semiconductor (MIS) allows quantifying the number of defects $\left(N_{D}\right)$ in the insulator bulk as well as at the insulator/semiconductor interface [15]. In the field of photovoltaics (PV) the CV characterization method is commonly used to analyze materials for surface passivation of crystalline silicon (c-Si). Several publications have discussed this method for characterization of passivating dielectric films like aluminum oxide $\left(\mathrm{Al}_{2} \mathrm{O}_{3}\right)[6$, 7] silicon nitride $\left(\mathrm{SiN}_{\mathrm{x}}\right)[5,8]$ and intrinsic and hydrogenated amorphous silicon ((i) a-Si:H) films [5, 9].

More information about the method and technology of $\mathrm{CV}$ spectroscopy itself are also given in the above mentioned references [1-5].

The band gap of $\mathrm{Al}_{2} \mathrm{O}_{3}(\sim 8 \mathrm{eV})$ as well as of $\mathrm{SiN}_{\mathrm{x}}$ $(\sim 4.5 \mathrm{eV})$ is very large, leading to the classification of these materials as insulators $[1,10,11]$. In contrast (i) a$\mathrm{Si}: \mathrm{H}$ is a semiconductor with a band gap of $\sim 1.7 \mathrm{eV}$ [5].

The state of the art method to determine $N_{D}$ by $\mathrm{CV}$ spectroscopy is based on Eq. (1) $[1,3]$ by considering the elementary electric charge (q), the measured capacitance value in case of accumulation $\left(C_{a}\right)$, the measured capaci-

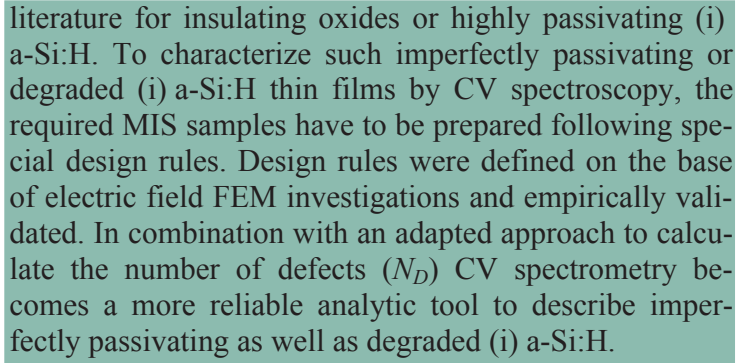

tance value at low frequency $\left(C_{L F}\right)$ and the capacitance value at high frequency $\left(C_{H F}\right)$.

$$
N_{D}=\frac{\int C_{a} \cdot\left(\frac{C_{L F} / C_{a}}{1-C_{L F} / C_{a}}-\frac{C_{H F} / C_{a}}{1-C_{H F} / C_{a}}\right) d V}{q} .
$$

$C_{a}$ has to be measured under forward-bias conditions while $C_{L F}$ and $C_{H F}$ are measured under reverse-bias conditions. Further it has to be mentioned, that a MIS structure containing a semiconductor like (i) a-Si:H behaves like a Schottky diode under reverse-bias CV measurement conditions $[1,3]$.

The determination of $N_{D}$ by solving Eq. (1) works best for MIS structures containing insulator materials like $\mathrm{Al}_{2} \mathrm{O}_{3}$ or $\mathrm{SiN}_{\mathrm{x}}[6,8]$. The determination of $N_{D}$ of (i) a-Si:H containing MIS structures requires an excellent passivation quality of the (i) a-Si:H layer.

Imperfectly passivating or thermal or light-induced degraded (i) a-Si:H exhibits a reduced passivation quality as well as an increased defect based conductivity [12-14]. In consequence, the classical approach of CV spectroscopy as 
described before by using Eq. (1) is not sufficient for those CV MIS samples.

In more detail, the avalanche voltage of imperfectly passivating or degraded (i) a-Si:H is influenced by a defect induced shunt conductivity. Therefore, it is not possible to determine $C_{a}$ in forward bias. Further the defect induced conductivity yields lateral bias as well as frequency dependent shunt conductance effects.

As a consequence, a new approach for determination of $N_{D}$ on the base of CV spectroscopy analysis is necessary, as well as an adaption of the CV MIS sample structure itself.

2 Experimental details Several samples of (i) a-Si:H containing MIS structures as well as standard lifetime reference samples were prepared.

The overall number of defects $\left(N_{D}\right)$ was analyzed after deposition and thermal activation $\left(4 \mathrm{~min} / 200^{\circ} \mathrm{C}\right)$ as well as during thermal treatment for up to $100 \mathrm{~h}$. Thermal treatment was carried out using a hotplate in ambient air and in the dark at a temperature of $200 \pm 2^{\circ} \mathrm{C}$.

Calculating $N_{D}$ based on of $\mathrm{CV}$ data obtained with a HP 4192A impedance analyzer was performed as described in Section 4. $N_{D}$ reference values were calculated using the Olibet model [15]. The model itself is based on the effective minority carrier lifetime $\left(\tau_{e f f}\right)$ measurements [15]. Related measurements were performed using a WCT 120 lifetime tester from Sinton Instruments [16].

2.1 Sample preparation All samples discussed in this study were based on plasma enhanced chemical vapor (PECV) deposited (i) a-Si:H with a columnar morphology [17-19]. A columnar morphology was chosen as investigations pointed out that there is an appreciable decrease in the passivation quality of columnar grown (i) a-Si:H during thermal treatment $[18,19]$.

$\mathrm{CV}$ as well as lifetime samples were processed on phosphorous doped (n-type, $5 \Omega \mathrm{cm}, 250 \mu \mathrm{m},<100>$ oriented) RCA cleaned float zone (FZ) silicon wafers with a chemically polished surface.

Lifetime samples consisted of $50 \times 50 \mathrm{~mm}^{2} \mathrm{c}-\mathrm{Si}$ substrates passivated by $40 \mathrm{~nm}$ columnar (i) a-Si:H. Deposition was carried out in a PlasmaLab 100 direct PECVD reactor from Oxford Instruments at a temperature of $225^{\circ} \mathrm{C}$ [20].

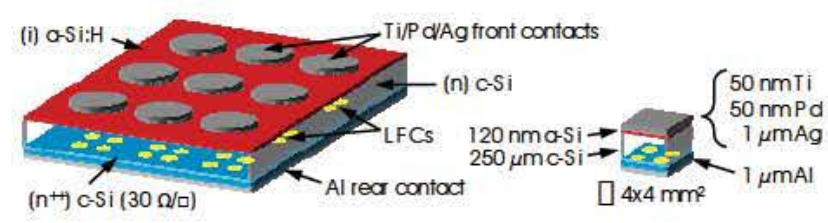

Figure 1 Schematic of the CV MIS sample structure in the classic design (left) as well as in shaped design (right). Marked and named properties and layers are the same for both designs. Unscaled illustration.
Remaining native silicon oxide was removed in diluted HF prior to deposition.

Preparing CV samples required considerably larger preparation efforts due to their complexity, Fig. 1. The preparation employed standard processes and was similar to the process flow discussed in [20].

At the interface between the rear side contact and the c-Si carrier substrate a $30 \Omega / \square\left(n^{++} / n\right)$ high/low junction was established to form an ohmic like contact and prevent the formation of a Schottky contact. Tube furnace $\mathrm{POCl}_{3}$-based diffusion in combination with further processes as well as wet chemical etching steps had to be performed implementing this high/low junction. More details of these standard preparation techniques are given in [20].

The rear contact of the CV samples consists of $1 \mu \mathrm{m}$ thick, electron beam evaporated aluminum. Additional laser fired contacts (LFCs) on the rear side were established to minimize the ohmic contact resistance further, Fig. 1 [21].

Afterwards the front side of the $\mathrm{CV}$ samples was covered by a $120 \mathrm{~nm}$ thick columnar (i) a-Si:H layer by PECV deposition.

The final front contacts consist of a $\mathrm{Ti} / \mathrm{Pd} / \mathrm{Ag}$ stack $(50 \mathrm{~nm} / 50 \mathrm{~nm} / 1 \mu \mathrm{m})$ which was deposited by electron beam evaporation. It is important to note that the front electrode was deposited after thermal treatment. This order was chosen to allow characterization of the decrease in passivation quality of (i) a-Si:H due to the effusion of hydrogen out of the layer during thermal treatment [22]. Adding the front contact prior to thermal treatment could hamper the hydrogen effusion and affect the experimental results. Earlier investigations have shown that electron beam evaporation has no influence on the passivation quality of the here discussed columnar (i) a-Si:H [20].

\section{CV spectroscopy}

3.1 Analyzing classical CV MIS structures The standard design for CV MIS structures consists of a large c-Si carrier substrate with a full sized rear contact and smaller front contacts on top of the insulator, Fig. 1 (left) $[5,7]$.

Preparing and testing such a classic structure including imperfectly passivating (i) a-Si:H leads to $\mathrm{CV}$ spectrometry results as shown in Fig. 2.

The capacitance was measured at $20^{\circ} \mathrm{C}$ in the dark in reverse-bias direction $(-7 \mathrm{~V} \ldots 0 \mathrm{~V})$. The bias is complemented by a superimposed low-level AC signal with an amplitude of $30 \mathrm{mV}$. Measurements were conducted at several frequencies of the $\mathrm{AC}$ signal between $10 \mathrm{kHz}$ and $1 \mathrm{MHz}$. The given $\mathrm{CV}$ measured capacitance in Fig. 2 is normalized to the area of the front contact metallization $\left(4 \pi \mathrm{mm}^{2}\right)$.

DC reverse-bias voltage affects the width of the spacecharge region while the $\mathrm{AC}$ voltage leads to a charge variation $[1,5]$. Electrons are trapped and emitted by defects in the (i) a-Si:H bulk as well as at the (i) a-Si:H/c-Si hetero- 
junction. These charges contribute to the overall capacitance and can be measured. Due to the emission energy $\left(E_{e}\right)$ of charge carriers trapped in defects and with respect to the energetic distance of traps to the band edge the contribution of the trapped charge to the overall capacitance becomes frequency dependent [23]. As $E_{e}$ is large for low frequencies increasing the frequency of the $\mathrm{AC}$ voltage leads to a decoupling and less contribution of trapped charge. Therefore, it is expected that the overall capacitance at low frequency is higher compared to the capacitance measured at high frequencies $[1,5]$.

In the case of the absence of bias or at low reverse-bias levels close to $0 \mathrm{~V}$ as well as in the case of depletion at high reverse-bias levels (abs. bias $\gg 0 \mathrm{~V}$ ), the contribution of the space charge region to the capacitance of the MIS structure is dominant. As a consequence, it is expected that the capacitance can become quasi-independent of the AC frequency in these cases. Moreover, the aforementioned Schottky diode like characteristic of the CV graphs are expected under reverse-bias direction $[1,5]$.

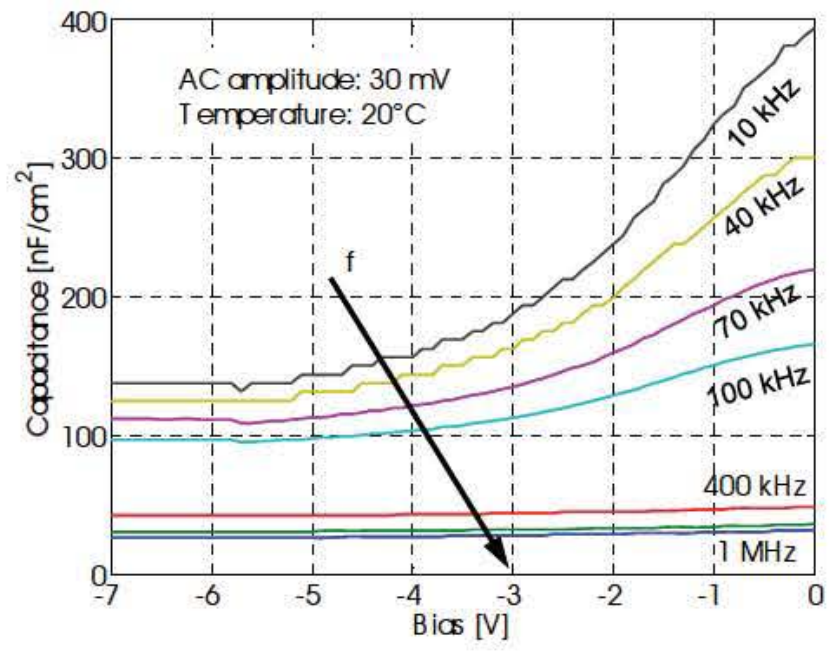

Figure $2 \mathrm{CV}$ spectroscopy of a classical (i) a-Si:H CV MIS sample in reverse as a function of the bias voltage for various frequencies of the superimposed low-level AC signal.

The CV plots displayed in Fig. 2 show the behavior of Schottky diodes. Also, the overall capacitance measured at an $\mathrm{AC}$ frequency of $10 \mathrm{kHz}$ is always higher than the one measured with a frequency of $1 \mathrm{MHz}$.

However, the capacitance at absolute bias $=0 \mathrm{~V}$ measured at $10 \mathrm{kHz}$ is more than eight times higher compared to the one measured at $1 \mathrm{MHz}$ and four times higher at $-7 \mathrm{~V}$.

This huge capacitance deviation between low and high frequency measurements can not only be attributed to defects in the (i) a-Si:H beneath the front contact. Moreover, it can be assumed that defects in the (i) a-Si:H layer beside the front contact trap electrons leading to a lateral shunt conductance.

\subsection{Adapted design for CV MIS samples}

A numerical simulation of the electric field beneath and beside the front contact of a classic CV MIS structure is shown in Fig. 3 (left). Simulations were done by solving Laplace's equation using the FEM-tool FlexPDE.

The simulation supports the aforementioned assumption of shunt conductivity induced by traps in the classic MIS samples. As can be seen in Fig. 3 (left), the field strength in the amorphous structure as in the c-Si carrier substrate beside the front contact is comparatively high. Defects like dangling bonds located in this surrounding area of the front contact can easily contribute to the shunt conductivity induced by traps in the (i) a-Si:H.

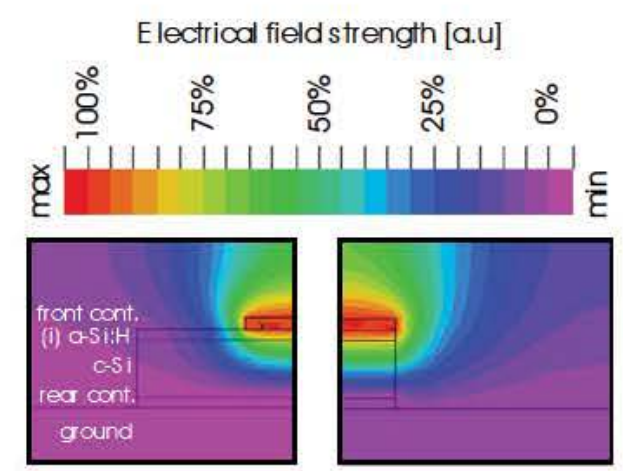

Figure 3 Electrical field strength FEM simulations of a classic CV MIS sample (left) and a separated CV MIS sample (right). Simulated samples according to the design and geometries given in Fig. 1 and surrounded by air. Unscaled illustration.

Furthermore, it can be assumed from the measurement in Fig. 2 that the effect of a trap based conductance is frequency dependent too. In consequence the effective area of the front contact as well as the permittivity $\left(\varepsilon_{r}\right)$ becomes frequency dependent.

A hands-on approach to address these challenges is the processing of separated CV MIS samples, see Fig. 1 (right). Separating samples with full size metal front as well as back electrodes by using a dicing saw yields quadratic samples. The FEM simulation of the electrical field strength of this adapted design is displayed in Fig. 3 (right). As a result of the geometric constrains, the electric field distribution within the sample is more homogeneous compared to the classical design.

For the sake of completeness it should be mentioned that a quadratic shape of $\mathrm{CV}$ samples is less ideal than a round shape because of corner effects. However, the results displayed and discussed in the following Section 3.3 suggest that the influence of the quadratic shape on the samples investigated in this study is negligible.

3.3 Analyzing adapted CV samples Figure 4 shows a set of CV spectroscopy measurements of an adapted MIS sample according to Fig. 1 (right). The range of the displayed reverse bias in Fig. 4 is between $-1.8 \mathrm{~V}$ to $0 \mathrm{~V}$. The frequency of the superimposed $30 \mathrm{mV}$ amplitude 
signal changes in $10 \mathrm{kHz}$ steps from $100 \mathrm{kHz}$ up to $200 \mathrm{kHz}$.

The shapes of the line plots strongly differ from those given in Fig. 2. The removal of (i) a-Si:H beside the front contact of separated CV MIS samples and the related absence of lateral trap-induced conductivity improve the quality of the CV spectroscopy results. The measured CV graphs fit to the expected shape of a Schottky diode. Furthermore, the capacitances at low as well as high bias voltages become independent of the AC frequency.

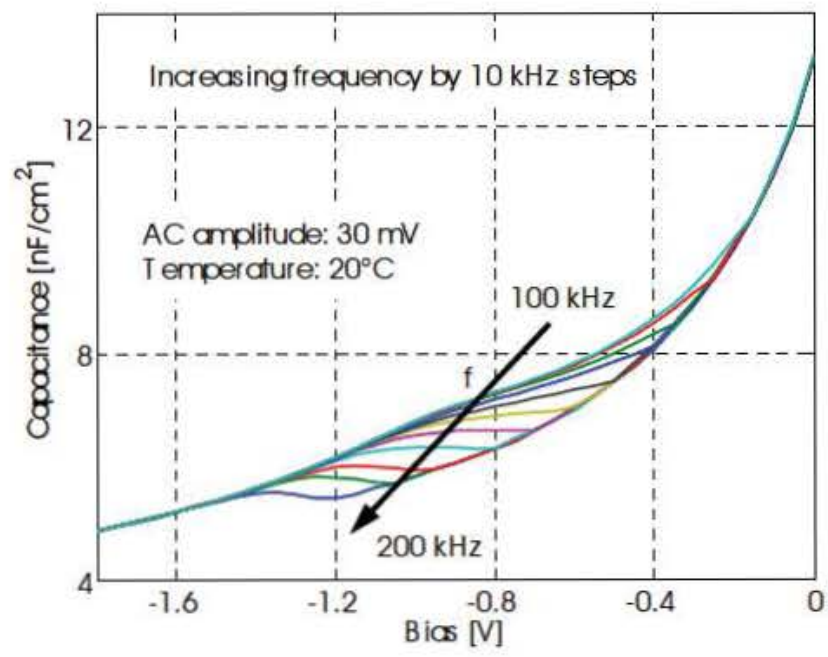

Figure $4 \mathrm{CV}$ spectroscopy of an adapted (i) a-Si:H CV MIS sample in reverse as a function of the bias voltage for various frequencies of the superimposed low-level AC signal.

However, the standard approach to determine the overall number of defects $\left(N_{D}\right)$ as given in Eq. (1) is still not applicable to these adapted CV MIS samples including imperfectly passivating (i) a-Si:H. The necessary parameter $C_{a}$ cannot be determined due to the trap based conductivity in forward bias.

\section{Improved calculation of $N_{D}$ and validation}

4.1 Calculation of $N_{D}$ The comparison of $C V$ spectrums at low frequency and high frequency shows an elliptical gap between the curves, Fig. 4 . The total charge related to this elliptical area can be attributed to the contribution of electrons trapped in defects. Furthermore, as the Schottky diode becomes conductive in forward bias the theoretical value of $C_{a}$ is infimum. Based on this assumption the calculation of $N_{D}$ can be derived from Eq. (1) as given in Eq. (2).

$$
N_{D}=\frac{\int\left(C_{L F}-C_{H F}\right) \cdot d V}{q} .
$$

The calculation considers in addition to the elementary electric charge (q) the difference of the capacities measured at low and high frequencies as well as the potential width of the elliptical capacitance gap $(\mathrm{d} V)$.
4.2 Validation The lifetime reference samples were prepared and treated as described in Section 2. $N_{D}$ values were calculated based on effective minority carrier lifetime measurements $\left(\tau_{e f f}\right)$ by using the Olibet model [15].

Figure 5 shows measured values of $\tau_{\text {eff }}$ (red circles) directly after deposition and thermal activation as well as after additional thermal treatment for $1 \mathrm{~h}, 10 \mathrm{~h}$ and $100 \mathrm{~h} . \tau_{\text {eff }}$ is determined at an injection level of $\Delta n=1 \times 10^{15} \mathrm{~cm}^{-3}$.

As can be seen in Fig. 5, the passivation quality of the columnar (i) a-Si:H layer remains stable after the first hour of thermal treatment, as $\tau_{\text {eff }}$ remains at $\sim 3.3 \mathrm{~ms}$ within measurement accuracy.

This value of $\tau_{e f f}$ is relatively low and indicates that the investigated columnar (i) a-Si:H is just imperfectly passivating. Performing thermal treatment $\tau_{e f f}$ decreases as described in [18] and is found to be $>1 \mathrm{~ms}$ after $100 \mathrm{~h}$. Due to the thermal treatment, hydrogen effuses out of the layer, leaving behind unsaturated dangling bonds which act as defects limiting minority carrier lifetime.

Furthermore, Fig. 5 shows the related number of defects in the (i) a-Si:H as well as its changes with thermal treatment. Compared are the values of $N_{D}$ calculated using Eq. (2) and based on CV spectroscopic analysis (blue diamonds) to the reference values of $N_{D}$ calculated using the Olibet model (black squares). In either case, the decrease of $\tau_{\text {eff }}$ coincides with an increase of $N_{D}$. This is related to the aforementioned effusion of hydrogen out of the layer $[18,22]$.

Comparing the two different approaches to determine $N_{D}$ in Fig. 5, we observe a good qualitative and fair quantitative agreement which supports the validity of the presented approach. The parallelism of the two line plots points out that the proposed approach to determine $N_{D}$ from CV spectroscopy measurements by Eq. (2) is valid and can be applied to the study of thermally aged samples.

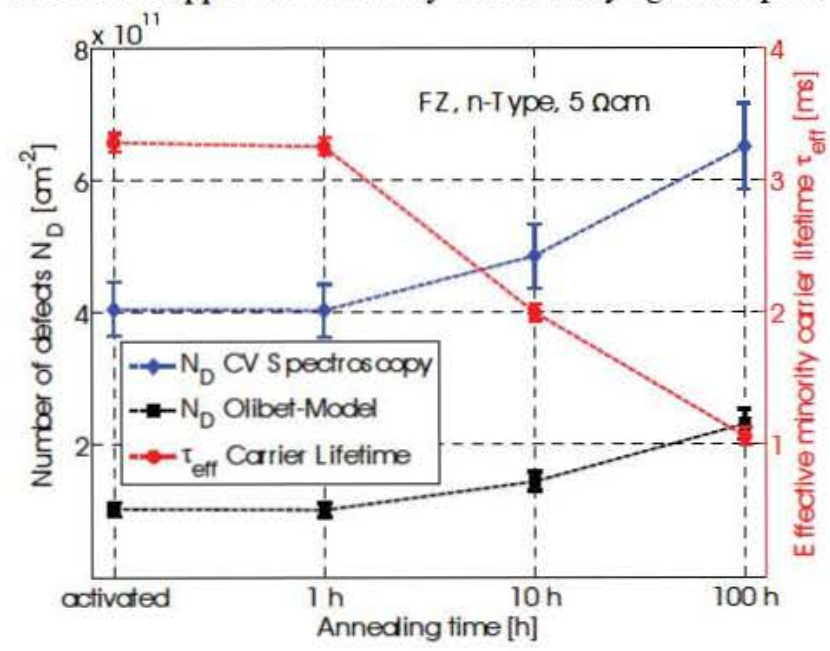

Figure 5 Comparison of $\mathrm{CV}$ spectrometry and Olibet model for the determination of the number of defects $\left(N_{D}\right)$ in columnar (i) a$\mathrm{Si}: \mathrm{H}$ as well as related values of the effective minority carrier lifetime $\left(\tau_{\text {eff }}\right)$. Lines are guides to the eye. 
The discrepancy in the determined number of defects can be partly explained by different sample dimensions. The investigated CV MIS structures exhibits a perimeter to surface ratio of $1: 2 \mathrm{~m}^{-1}$ while the ratio for the $\tau_{\text {eff }}$ samples is $1: 25 \mathrm{~m}^{-1}$. In consequence, edge effects such as defects account for a higher contribution to the overall capacitance of the smaller CV MIS samples.

Furthermore, a contribution of the different methods and their different specific sensitivities is supposable. The method determining $N_{D}$ on base of $\tau_{\text {eff }}$ measurements by using the Olibet model is very sensitive to defects at the (i) a-Si:H/c-Si interface. Most of the minority carriers recombine at dangling bonds in the immediate vicinity to the interface. In contrast to this, $\mathrm{CV}$ spectroscopy takes into account all defects between the electrodes of the MIS samples for the determination of $N_{D}$. Therefore, the CV spectroscopic approach is more sensitive to defects in the bulk of the (i) a-Si:H layer than the approach using the Olibet model.

Aging of (i) a-Si:H, like occur by thermal treatment, leads to an effusion of hydrogen out of the layer. The hydrogen leaving defects like dangling bonds behind. The parallelism of the both compared methods to determine $N_{D}$, shown in Fig. 5, displays that the aging occurs all over the (i) a$\mathrm{Si}: \mathrm{H}$ film in the same extend.

5 Conclusions The adapted design rule of full area samples including full area front and rear contacts leads to a physical confinement of the electric fields in CV MIS samples. This new approach allows to characterize imperfectly passivating as well as degraded (i) a-Si:H with a high shunt conductivity by CV spectroscopy analysis.

The defect induced shunt conductivity inhibits the determination of the overall accumulated capacity in forward-bias. As a consequence, an alternative method of calculating the number of defects out of $\mathrm{CV}$ spectroscopy analysis was discussed. The calculation of $N_{D}$ only from reverse-bias CV spectroscopy has been introduced, discussed and validated. Validation itself was based on the comparison with $N_{D}$ reference values determined by using the Olibet model and based on $\tau_{\text {eff }}$ measurements. The effect of thermal treatment on $N_{D}$ was determined using the new method and compared to the reference protocol. Results show that the new approach calculating $N_{D}$ out of CV data is valid. Differences in the total number of $N_{D}$ can be ascribed to different sample sizes as well as to differences in the sensitivity of the methods.

Acknowledgements Part of this work was supported by the German Federal Ministry for Economic Affairs and Energy (FKZ 0325581). The content is the responsibility of the authors.

\section{References}

[1] D. K. Schroder, Semiconductor Material and Device Characterization (John Wiley \& Sons, Inc, New Jersey, 2006).
[2] E. H. Nicollian and J. R. Brews, MOS (Metal Oxide Semiconductor) Physics and Technology (John Wiley \& Sons, New York, 2002).

[3] M. Kuhn, Solid State Electron. 13, 873 (1970).

[4] T. Walter, R. Herberholz, C. Müller, and H. W. Schock, J. Appl. Phys. 80, 4411 (1996).

[5] W. G. J. H. M. van Sark, L. Korte, and F. Roca, Physics and Technology of Amorphous-Crystalline Heterostructure Silicon Solar Cells (Springer-Verlag, Berlin, 2012).

[6] H. Goverde, B. Vermang, A. Morato, J. John, J. Horzel, G. Meneghesso, and J. Poortmans, Energy Procedia 27, 355 (2012).

[7] T. Lüder, Passivierung von kristallinem Silizium mit Aluminiumoxid-Dünnfilmen für Solarzellenanwendungen, $\mathrm{PhD}$ Thesis, University of Konstanz, Konstanz, 2014.

[8] S. Joos, Y. Schiele, B. Terheiden, H.-W. Becker, D. Rogalla, and G. Hahn, Energy Procedia 55, 786 (2014).

[9] A. S. Gudovskikh, J.-P. Kleider, and E. I. Terukov, Semiconductors 39, 940 (2005).

[10] R. H. French, J. Am. Ceram. Soc. 73, 477 (1990).

[11] S. V. Deshpand and E. Gulari, J. Appl. Phys. 77, 6534 (1995).

[12] H. Plagwitz, B. Terheiden, and R. Brendel, J. Appl. Phys. 103, 094506 (2008).

[13] L. Serenelli, R. Chierchia, M. Izzi, M. Tucci, L. Martini, D. Caputo, R. Asquini, and G. de Cesare, Energy Procedia 60, 102 (2014)

[14] R. A. Street, R. W. Cahn, E. A. Davis, and I. M. Ward, Hydrogenated Amorphous Silicon (Cambridge University Press, Cambridge, 1991).

[15] S. Olibet, E. Vallat-Sauvain, and C. Ballif, Phys. Rev. B 76, 035326 (2007).

[16] R. A. Sinton and A. Cuevas, Appl. Phys. Lett. 69, 2510 (1996).

[17] J. C. Knights and R. A. Lujan, Appl. Phys. Lett. 39, 244, (1979).

[18] S. Gerke, H.-W. Becker, D. Rogalla, G. Hahn, R. Job, and B. Terheiden, in: Proc. 29th EU PVSEC, Amsterdam, Netherlands, 2014, pp. 9-12.

[19] S. Gerke, H.-W. Becker, D. Rogalla, G. Hahn, R. Job, and B. Terheiden, Energy Procedia 77, 791 (2015).

[20] S. Gerke, A. Herguth, N. Brinkmann, G. Hahn, and R. Job, in: Proc. 28th EU PVSEC, Paris, France, 2013, pp. 26002603.

[21] E. Schneiderlöchner, R. Preu, R. Lüdemann, and S. W. Glunz, Prog. Photovolt.: Res. Appl. 10, 29 (2002).

[22] R. A. Street, C. C. Tsai, J. Kakalios, and W. B. Jackson, Philos. Mag. B 56, 305 (1987).

[23] J. T. Heath and J. D. Cohen, J. Appl. Phys. 95, 1000 (2004). 